. 


\title{
THE NBS VISION SYSTEM \\ IN THE AMRF
}

\author{
Marilyn Nashman \\ Karen J. Chaconas
}

December 1987

Certain commercial equipment, instruments, or materials are identified in this paper in order to adequately specify the experimental procedure. Such identification does not imply recommendation or endorsement by the National Bureau of Standards, nor does it imply that the materials or equipment identified are necessarily the best available for the purpose.

This publication was prepared by United states Government employees as part of their official duties and is, therefore, a work of the U.S. Government and not subject to copyright. 



\section{TABLE OF CONTENTS}

I. Introduction I

II. System Overview 2

1. Vision and the Real-Time Control System 2

2. Vision and the Material Handing Station 2

III. Architecture Description 4

1. First stage Vision (FSV) 4

2. Second Stage Vision A (SSVA) 5

3. Second stage Vision B (SSVB) 6

4. Multilevel Database and Server (MLDSERV) 8

5. Supervisor (SUP) 11

6. Netboard (NET) 12

7. Physical Description of the Vision System 13

IV. System Operation 15

V. System Interface 19

VI. Future Plans $\quad 22$

VII. References 23

Appendix A. Data Structures 24

Appendix B. Interactive Debugging Features 43 


\section{IIST OF FIGURES}

Figure 1. AMRF Shop Floor

Figure 2. Enlargement of the Vision System Work Area Figure 3. NBS Vision System 
This document describes the NBS Vision System in the AMRF. It discusses the objectives of the vision system and its applications in the factory environment. Since the vision system is a multi-processor system, each process is described according to its position in the vision hierarchy as well as to its particular logical and computational functions. The unique hardware used is discussed and its capabilities described. In addition, a guide to operations is included: this contains step-by-step directions for "bringing up" the system in either stand-alone mode or integrated mode. The interfaces between the individual processes of the vision system, as well as the interfaces between the vision system and other AMRF systems, are described. Finally, appendices are included which describe data structures, and debugging features.

The document may be referenced at different levels. Section II describes the vision system in very general terms; it is intended for the reader who requires only an overview of the function of vision in the AMRF. Sections III and $V$ contain a more detailed description of the vision system and its interfaces with other systems. It assumes that the reader is familiar with computers and the concept of hierarchical design. A background in image processing techniques is useful in understanding the subroutines described. Section IV serves as an operations manual and is intended for the user who will be "bringing up" and monitoring the vision system. It assumes that the user is familiar with computers.

AMRF documentation packages describing the Horizontal Workstation and the Material Handing Workstation are useful references when reading this document. 
The vision system developed by the Sensory-Interactive Robotics Group of the Robot Systems Division at NBS serves two functions in the AMRF: it is used both by the Horizontal workstation (HWS) and the Material Handling workstation (MHWS). In both AMRF applications, the vision system processes an image only upon request. However it is constantly polling for command requests and monitoring the status of individual vision processing modules.

\section{VISION AND THE REAL-TIME CONTROL SYSTEM (RCS)}

There are currently three commands defined in the interface between the vision system and the RCS. Upon receiving an RCS command, vision first determines which information is being requested. Only information relevant to the type of question being asked is returned. Question one is a request for the centroid of the object in view; this enables RCS to center the robot tool over the part. Question two is a request for verification of a specific part, and if confirmed, the position and orientation of that part. Question three is a request for the computed range to the surface of the object in view. The answers supplied by the vision system enable RCS to compute the required robot motion to pick up the part at the ideal grasp point.

The vision system always supplies an answer to RCS after a request is made. The answer includes not only the requested information but a status flag which indicates success or failure of the action. A failed action can be the result of non-recognition of an expected part or a result of a "poor" image. Causes of poor images include improper lighting conditions, camera exposure problems, or degraded camera images. As will be explained further, the vision system attempts to adjust its internal parameters to overcome failures caused by poor images. An action is considered to be successfui if an expected part can be favorably matched to its internal model (see description of vision process MLDSERV), and if that part is located in the approximate portion of the tray in which it had been predicted.

\section{VISION AND THE MATERIAI HANDLING WORKSTATION (MHWS)}

The vision system acts to verify the contents of trays for the Material Handing workstation. There is one command defined in the vision system's interface with MHWS. This question requests 
verification, position, and orientation of parts on a tray. At present, there is only one configuration of the tray that can be verified. This request can be initiated either by an MHWS network communication or by a manually triggered signal sent from a remote location. It is expected that the methods used in the default verification can be expanded to be used on any tray configuration when actual MHWS data is entered into the AMPF database. At that time, the vision system will request a tray definition report and a tray contents report and will extract the pertinent information required for it to verify the identity and position of the expected parts. The interface between the vision system and the AMRF database has been independently tested successfully. At the completion of the verification task, vision sends a status report to MHWS. Currently, this report consists of a "done" flag, but in the future will include fields indicating success or failure of the task as well as updated positions and orientations of the verified parts. 


\section{ARCHITECTURE DESCRIPTION}

The vision system is designed in a hierarchical manner: commands from the control system and/or the Mtwis are decomposed into lower level tasks and executed by the appropriate process. There are currently six independent processes, operating asynchronously, that analyze and extract information from an image scene. Each process resides on its own microprocessor board and communicates to other processes via a pre-defined memory block (common memory). System routines have been developed to insure the integrity of this data transfer.

Currently, three of the processes operate in a bottom-up mode to read an image and globaliy extract information from it. These processes are First Stage Vision (FSV), Second Stage Vision A (SSVA) and second stage Vision B (SSVB). The Multi-Level Database Processor (MLDSERV) acts in a top-down mode using its model database to identify specific objects in a scene. The supervisor (SUP) process monitors vision system activity and communicates with the "outside world" via the Netboard (NET) processor (used for communicating with MHWS and the AMRF Database) or a 589 board 1 (used for communicating with the RCS). Requests for information are passed from SUP to the server which resides on the same board as MIDSERV. A more detailed description of the functions of each process is presented below.

\section{FIRST STAGE VISION (FSV)}

First Stage Vision is the lowest level in the vision processing hierarchy; it acts as the interface between the camera hardware and the upper levels of the vision system. It is responsible for receiving requests for image information and translating those requests to the camera hardware. It is further responsible for reading back run-length data and transferring it to the appropriate common-memory locations for the requesting processes.

FSV controls the commands sent to the Digital Analog Design (DAD) Frame Buffer which reads in an image and converts it to a binary image according to a threshold value supplied by sup. The binary image is converted to run-length encoded information by DAD and is read back by FSV.

In addition to collecting data for other processes, FSV can also act as a fast ranging mechanism. When appropriately commanded by the vision supervisor module, it can read in an image and compute the range to the closest point in a given windowed area in real time. 
NBS Vision System

FSV is coded in $C$ and assembly language. The code was developed on a CPM S100 system and is downloaded to an 8086 microprocessor.

\section{SECOND STAGE VISION A (SSVA)}

SSVA receives commands from second stage Vision B (SSVB), and passes them down to FSV. It then waits for FSV to read in an image and pass the run-length encoded data back to SSVA. SSVA performs different operations on the resulting data depending on the kind of picture (flood or structured light) being processed.

on receiving picture data from a floodlit image, SSVA performs a connected-components analysis and constructs a tree of the objects (blobs) in the image. It also computes various mass properties of each blob (area, centroid, and moments) and extracts the boundary. This structure is passed to SSVB.

When the image results from using structured light, SSVA constructs groups of connected curve segments which will be described by SSVB in terms of Chebyshev polynomials. The segments correspond to single curves in the image or to pieces of curves, starting where a curve spiits and ending when it ends or when it merges with another curve. The structures are passed up to SSVB, and SSVA polls SSVB until it receives the next command.

The following files contain the code used to implement the actions of SSVA:

ssva.h This file contains default parameters and constants, as well as descriptions of the structures used to store objects and to pass commands.

ssva.cm This file, along with vbus.h, are files that define operating system parameters and addresses particular to the individual processor. In general, they are included in other files but are only modified by the operating system.

ainit560.c This code performs the hardware-dependent

initializations. Currently these include setting camera variables and operating system parameters.

ssva.c This is the main program. It polis SSVB for commands, sends the commands to FSV, and waits for the image to be returned. It then invokes 
conn[12].C These routines implement the connected-components procedure. They perform the main processing for flood images, constructing the tree structure of blobs in the image and computing properties of each blob. It requires some assembly language subroutines.

interface.c This program carries out the communications with FSV. It reformats the commands received from SSVB, sends them to FSV, and then waits for the results of the processing performed by FSV.

isicheb.c

This file and the two described below contain the algorithms for segmenting curves for Chebyshev processing. Inicheb.c initializes the line-linking process for structured-light images on a row-by-row basis.

Funlen.c

This file contains the algorithm that computes the extent of each curve.

split.c

This program performs some postprocessing to split curves at points of sharp orientation change, i.e. corners.

\section{SECOND STAGE VISION B (SSVB)}

SSVB receives commands from the first level of the Multilevel Database Processor (MLDSERV) and passes them down to SSVA. It then waits for SSVA to perform connected components analysis or curve segmentation and construct a tree of the objects (blobs) or curves in the image. SSVB accepts the tree of structures and performs feature analysis on each object in the image. For floodlit images, this currently involves finding the corners, principal axis, number of holes, and perimeter of each object. For structured-light images, each segment is described in terms of a polynomial, and the endpoints are reported as features. SSVB sends the tree of components and a set of structures describing the features to MIDSERV and then polls until it receives the next command from MIDSERV.

The following files contain the code used to implement the 
action of SSVB:

ssvb.h

ssvb.cm

init $560 . c$

ssvb.c

readssva.c

cornfast.c

cornslow.c
This file contains default parameters and constants, as well as descriptions of the structures used to store objects and features and to pass commands.

This file and vbus.h are files that define operating system parameters and addresses particular to the individual processor. In general, they are included in other files but only modified by the operating system manager.

This code performs the hardware-dependent initializations. Currently these include setting camera variables and operating-system parameters. This file is maintained by the operating system manager. (The same program is used to initialize MLDSERV) .

This is the main program. It polls MLDSERV for commands, sends the commands to SSVA, and waits for the connected components structure or the curve segment structures to be returned. For flood images, it then invokes the various feature extraction procedures for each component and sets up structures describing the results. For structured-light images, Chebyshev polynomials are computed and segment endpoints found. The results are written out to MLDSERV. Error conditions are flagged and reported to SUP, and the program goes back to poll MLDSERV.

This program receives the tree of components or the curve segments from SSVA. It reformats the data into a more useful form for later processing.

This code finds the corners in the boundary of an object. Cornfast contains a fast algorithm that works on clean boundaries.

This routine finds the corners in the boundary of an object. Cornslow uses a k-curvature algorithm that takes longer than the algorithm in cornfast.c, but works better. A flag passed by the supervisor determines which algorithm is called on any particular image. 
prinax.c

numholes.c

perimeter.c

perimpma.a86

ssvbout.c

chebfit.c

chebcorn.c
This file computes the principal axis of an object.

This file finds the number of holes in an object. This file computes the perimeter of an object. This routine is an assembly-language version of the perimeter computation.

This routine writes the structures for objects and features or the Chebyshev structures to MLDSERV.

This routine computes the Chebyshev polynomials for each curve segment.

This routine finds the endpoints of Chebyshev segments and builds feature structures to describe them.

\section{MULTILEVEI DATABASE AND SERVER (MLDSERV)}

The purpose of MIDSERV is to store the results of the lower level processing in a structure appropriate for the upper level recognition algorithms. The server is the portion of MLDSERV which interprets commands received by the supervisor and attempts to answer the requests. Its main activities are concerned with recognizing objects and extracting relevant information from the database. MLDSERV also performs some of the range and position computations and is responsible for driving the graphic displays.

The following files contain the code used to implement the actions of MIDSERV.

camera.h.

midi.h

init $560 . c$
This file contains external declarations of the camera variables.

This file contains default parameters and constants, as well as structure definitions for objects, features and communications with sUP and the lower levels of the vision system.

This routine performs hardware dependent initializations. Currently these include setting camera variables used in the range computations and initializing various parameters. (The same 
NBS Vision System

mldserv.c

$\operatorname{mldi.c}$

readssvb.c

findeqns.c

newcal.c

display.c

gap.c

marlchp.c

drawraw.c program is used to initialize SSVB).

This is the main program. It polls sup for commands to take pictures, for questions to be answered, and for error messages to be displayed. Depending on the input from SUP, either a command is sent down to SSVB to take a picture, the server is invoked to answer a question, or a display routine is called to output an error message. On completion of the task, any errors are reported to the supervisor, and the polling is resumed.

This routine sends commards to SSVB, calls the routine to set up the entries for the results in the database and causes the 3D position computations to be invoked.

This program receives the components and features from SSVB. It uses a dynamic storage allocation technique to store the data in a database.

This routine performs analysis of line-flash images. First, it attempts to pair up line segments. When it succeeds, it uses triangulation to compute the surface equations, orientations, and positions of the implied surfaces. It works with either Chebyshev or "blob" representations of curves.

This routine contains the procedures that implement the camera calibration. The procedures allow transformations from image coordinates to real-world coordinates, and vice-versa (in a number of different coordinate systems).

This routine contains output routines for printing information about objects and displaying objects and features on a graphics display.

This set of routines contains low-level routines for driving the graphics display device.

This file contains low-level routines for driving the graphics chip.

This file contains routines for displaying the raw data read by $\mathrm{FSV}$. 
caption.c

malloc.c

server.c

reformat.c

fldmatch.c

marlch.a86

freeall.c

models.C

quest5.c

quick.c

sidelength.c
This routine supplies captions for the displays created by display.c and drawraw.c and the text describing the displays created by midserv.c and test.c.

This routine contains the dynamic storage allocations and freeing algorithms.

This is the main program for the questionanswering part of the system. It accepts questions and attempts to answer them by looking in the database. server.c contains the routines for answering questions involving range and orientation information and for answering guestions about the largest object in the field of view. It also contains many utility subroutines.

This routine contains procedures to reformat the questions asked by the control system into the structures used internally by the server and to reformat the answers into the structure required by the control system.

This routine contains the algorithms used to recognize parts from floodlit images (prismatic parts).

This is the driver for the Matrox graphics boards 1 .

This file contains routines that free dynamically allocated memory for the various structures.

This file is where the object models are defined and initialized.

This has the routine to begin processing the tray verification request received from MHW.

This contains the algorithm for answering fast flood questions and returning the position of the largest object in the field of view of the camera.

This routine performs the computations that compare the side lengths of sensed objects with the models and either accepts or rejects 


\section{matches on this basis.}

\section{SUPERVISOR (SUP)}

SUP is considered to be the "brains" of the vision system: it accepts commands from either RCS or MHWS, interprets them, translates them into a form suitable for the vision system, and initiates the command sequence in the vision system. If a poor quality image is detected, SUP tries to adjust whatever is necessary to obtain a good picture and then repeats the process. If there are no processing errors, SUP sends the appropriate status and answer to either RCS of MHWS. SimuItaneousIy, SUP checks that each of the vision levels is working. Furthermore, it monitors the SUP keyboard to service any of the interactive features described in Appendix B.

A short description of each of the files is given below:

super.h

talk.h

super.cm

sil.c

s12.c

sI3.c

s $14 . \mathrm{C}$

s15.c
This file defines constants and structures used by SUP.

This file defines constants and structures used to interface with the control system.

This file defines the common memory addresses of modules with which SUP communicates.

This is the main level routine. It contains global variable definitions, initializations, and the main loop. It is responsible for running the vision system and for error checking and compensation.

This section contains utility procedures for the command stack, procedures for getting new commands from the control system, procedures for starting a vision command, and procedures for sending answers back to RCS or MHWS.

This procedure reads and processes errors from ESV and SSVA.

This procedure reads and processes errors from SSVB and MIDSERV.

This routine contains the procedures which communicate with RCS through the 589 card. 
si6.c.

517.6

defaultt.c

makeparm.c

netport.a86

This file contains procedures for user interaction (operator service).

This file contains procedures for monitoring the vision command service.

This routine sets up question 5 for a default tray verification request.

This file sets up camera calibration parameters for the camera configuration in the AMRF.

This code interprets signals emitted by a remotely operated push button for generating an interactive tray verification request.

\section{NETBOARD (NET)}

The NET process acts as an interface between the vision system (SUP in particular), MHWS and the AMRF database. All functions contained in NET are activated by commands from SUP. These functions include database initialization requests, checking for MHW requests, and issuing requests for tray definition reports and tray contents reports. Communication between NET and either the AMRF database or MHWS is done over the AMRF network. A brief description of the routines resident on the NET board follows:

netbd.h

commur.h

address.h

net.cm

mbus.h

netmain.c
This is an "include" file containing definitions of structures and constants used by NET and SUP.

This is an "include" file describing the structure of command requests and status expected by MHWS as well as the structure of mailbox communications.

This file declares physical addresses used by NET in communicating with the AMRF database and MHWS.

This file contains vision system definitions of physical addresses used for inter process communications.

This "include" file contains the definitions of structures and constants used for maintaining inter process communications.

This is the main loop of the NET process. It 
initnet.c

This routine contains routines for starting the AMRF database communications: ABORT, INITIATE, and STARTUP.

netcommand.c This code polls on tray verification commands from MHWS. When one is received, it is read and the appropriate status is returned to SUP.

writenet.c

This file contains routines for generating status reports to MHWS upon completion of a tray verification sequence.

dbio.c

This routine constructs the status message to be returned to MHWS.

newmail.c

This file contains routines for reading and writing network mailbox communications.

netio.c This file is responsible for interprocess communication between SUP and NET.

The following routines are responsible for formatting or unpacking AMRF database communications: asnoi.c, userdsea.c. These routines use definitions provided by asn.h, asnapp.h, basic.h, imdassts.h, and userdse.h.

\section{PHYSICAI DESCRIPTION OF THE VISION SYSTEM:}

A General Electric Model 2500 camera is mounted on the T3 robot wrist in HWS, and a second camera is mounted on the gantry at the tray verification station. A flash box is attached to the robot mounted camera: this can be software activated to emit either a double planes of light flash or a point light flash. Double planes of light are used for determining range, pitch, and yaw of the object in view. The point light flash is used to compute the azimuth and roll of the object.

The image received from the camera is captured by a Digital Analog Design (DAD) Frame Buffer. The DAD hardware provides the ability to capture an image and threshold it in accordance with software provided values. The result of the thresholding operation is a binary image in which all grey level values greater than the supplied threshold are converted to white, while 
those values below the threshold are converted to black. The binary image is then compacted into run-length encoded data, i.e., only transitions from black to white or white to black are recorded. In addition to generating run-length encoded data, the DAD hardware also can filter "noisy" pixels from an image upon software command or can read and transfer a full grey scale image.

The vision system rack which is resident in the AMRF contains 8086 microprocessor boards for the vision processes as well as microprocessors for communication purposes. MLDSERV is executed from an 5100 board also located in the vision rack. 
IV. SYSTEM OPERATION

To start the vision system, locate the vision system rack which is to the right of the vision work area on the AMRF floor (see Figures 1 and 2). After opening the rear door of the rack, turn on the power strip to the left. Also, turn on the power strip on the right side of the three vision monitors that are in front of the glass window in the hallway. Plug in the lamp that is attached to the gantry at the tray verification station which is located in front of the Turning workstation.

At the vision work area, turn on the power strip to the right of the system monitors. To boot the s100 system, hit the carriage return key <CR> twice on the center keyboard. After the system has completed booting, the "H>" prompt will appear on the screen. If a system message followed by the system prompt does not appear within 10 seconds, toggle the switch labelled "Sloo Reset" on the switch box up and down. Then hit the carriage return key twice on the sloo monitor and wait for the "H>" prompt. TYpe "USER $10<C R>1$ on this same keyboard. Next, type "DOWNPARA - A ALIRI $G<C R>"$. The slo0 monitor will display comments about which process code it is downloading. The far right monitor, the Supervisor monitor, will display information concerning the status of the vision system start up and which processes are successfully running.

Two messages should appear next: one on the sioo monitor asking whether to enter in tray corner coordinates, and one on the Supervisor monitor asking for a <CR> when the network connections are present. The operator response to the tray corner coordinate query should always be "O" followed by <CR>. (Tray coordinates provide for the alignment of the lower left corner of the tray with the lower left corner of the image. They should be entered manually only if the tray position on the cart has changed significantly from past usage.) The prompt on the supervisor requires only a "<CR $"$ whenever the operator has verified that the vision system's network connections to MHWS and the database have been established. At the completion of the downloading procedure, the message "THE VISION SYSTEM IS UP!" will appear on the supervisor monitor. No further response is required of the operator. The vision system will run unattended until it is powered off.

Any variation on this sequence of events implies that the vision system is not running properly. The easiest remedy is to restart and redownload the vision processes. This can be done using the switchbox to the right of the vision system process monitors. Elip the lever marked "global" on the face of the box 


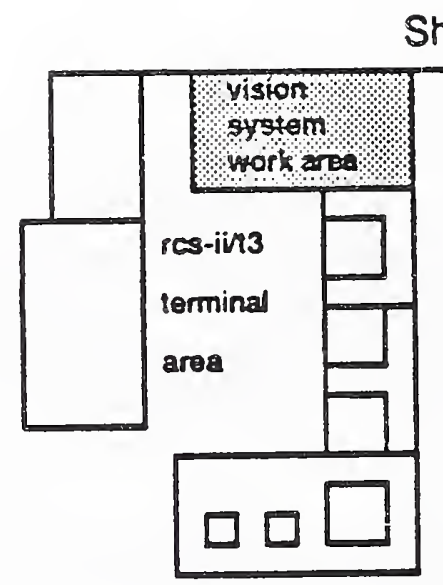

Shop Floor Barrier
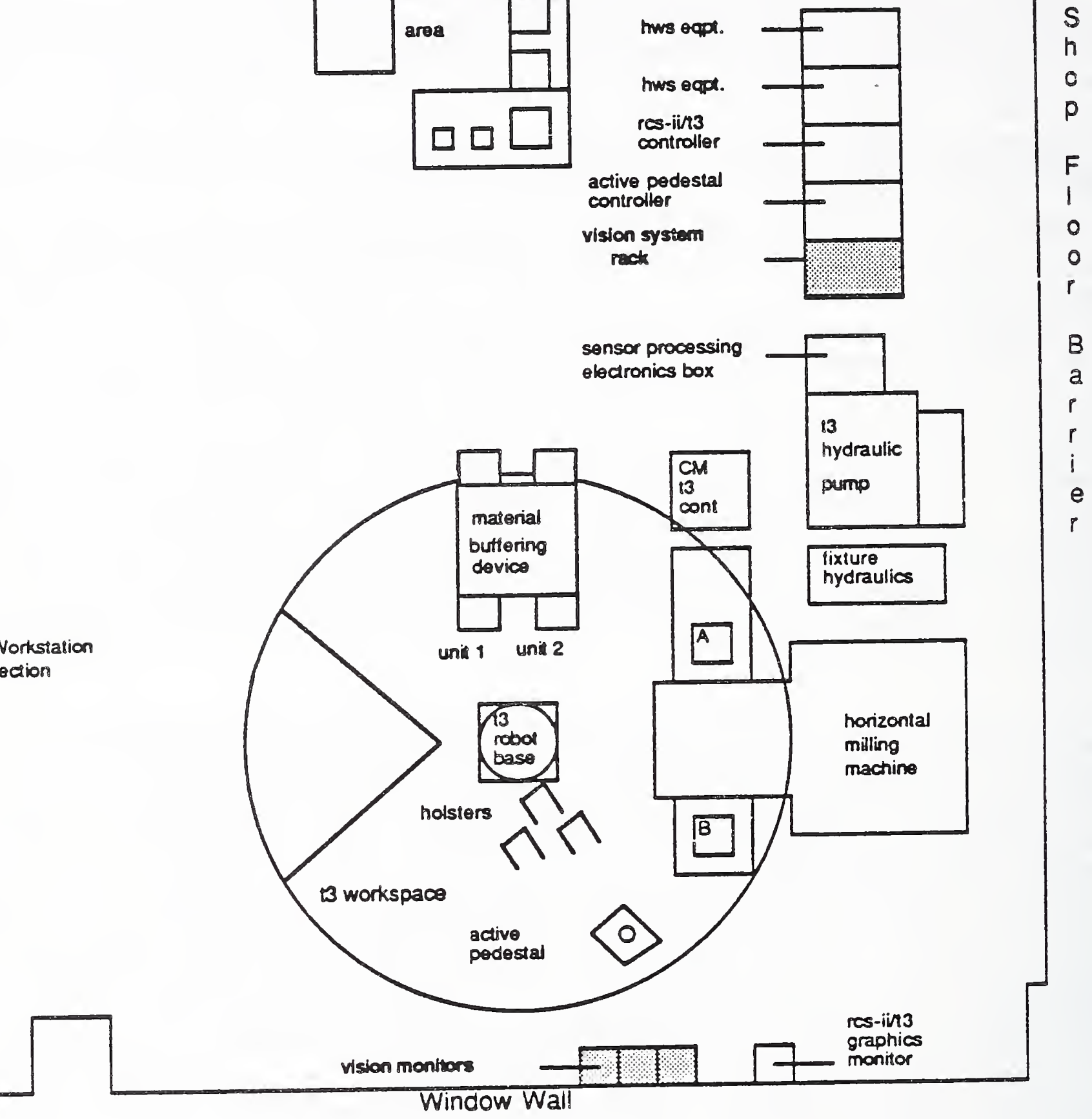

Figure 1. AMRF Shop Floor 


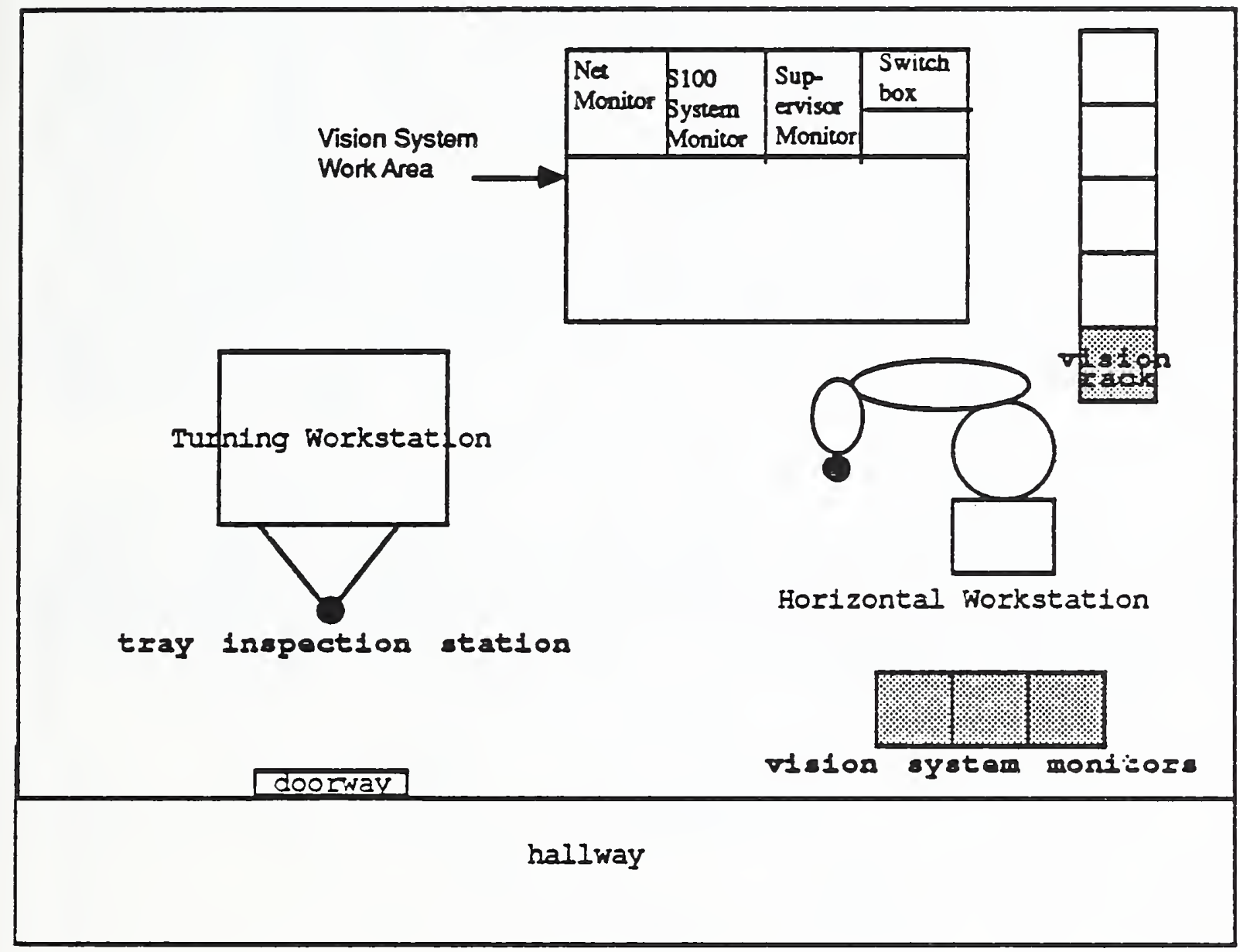

Figure 2. Enlargement of Vision System Work Area 
to the up position, then press the button marked "reset". Then restart the system by reentering "DOWNPARA - A ALLRI -G" on tine s100 keyboard.

The vision system has two modes of operation. During normal command mode, the vision system will poll and wait on commands from either RCS or MHWS (see section II). In simulated command mode, the operator can enter a command from the keyboard (see Appendix B). If the RCS is present and sends command "O", the vision system will generate its own simulated commands. The monitor on the left displays the status of the network process, NET, and will show the interaction of the vision system with MHWS. There are two other monitors that are used for displaying results of vision recognition tasks. The monitor on top of the SUp monitor displays the field of view that the current camera sees. The monitor on top of the sloo monitor is a graphic representation of the results of the most recent vision task.

To shut down the vision system, turn off the three power strips that are referred to in the initial power-up sequence and unplug the light above the tray inspection camera. close the doors on the vision system rack securely. 
The vision system processes communicate via a common memory area governed by a file system. The system operates in both a topdown and bottom-up manner (see Figure 2). The multiprocessing levels of the vision system pass raw data using a bottom-up process. FSV is responsible for commanding the camera to take a picture. The image data is passed to SSVA for processing and then to SSVB for further analysis. The multiprocessing levels perform object recognition in a top-down manner. SUP is responsible for starting and checking all levels of the vision system and for accepting commands from RCS. NET accepts commands from the Material Handling Work Station (MHWS). MIDSERV reformats these questions so that they may be passed down to the other processes.

The interprocess communications are handled using the vision system's MBUS library. The purpose of the MBUS Iibrary is to ensure data integrity of communications and to allow for asynchronous communications between processes. Blocks of predefined common memory, called "files", accessible to each vision process are defined. A set of flags associated with each file records its current state (i.e, which process owns the file, is it currently open or closed, which process accessed it last, and the system time of last access). Only those processes granted read and/or write permission can access a file. A user can only access a file if it is closed and must open the file before it can be either read or written. The concept of opening and closing files is analogous to the UNIX file system calls.

Communications betewen the RCS and the vision system are handled using a high speed parallel link. A master-slave relationship is represented on the board to describe the relationship between the two systems and to indicate the direction of communication. Communication is initialized by the RCS setting the appropriate bits. Communication protocol and a command structure are used to allow the RCS to request a command from the vision system (see Appendix A).

The MHWS and the vision system communicate using the VAX common memory mailbox system. Mailbox areas are established when the network communications are made to provide for message passing between the two systems and between the vision system and the AMRF database. When first brought up, the vision system initializes with the database by sending the UVA protocol commands, "ABORT", "INITIATE", and "STARTUP" [1]. Vision then polls the mailbox command area for a change in sequence number to detect when a tray verification request has been sent from MHW. 
NBS Vision System

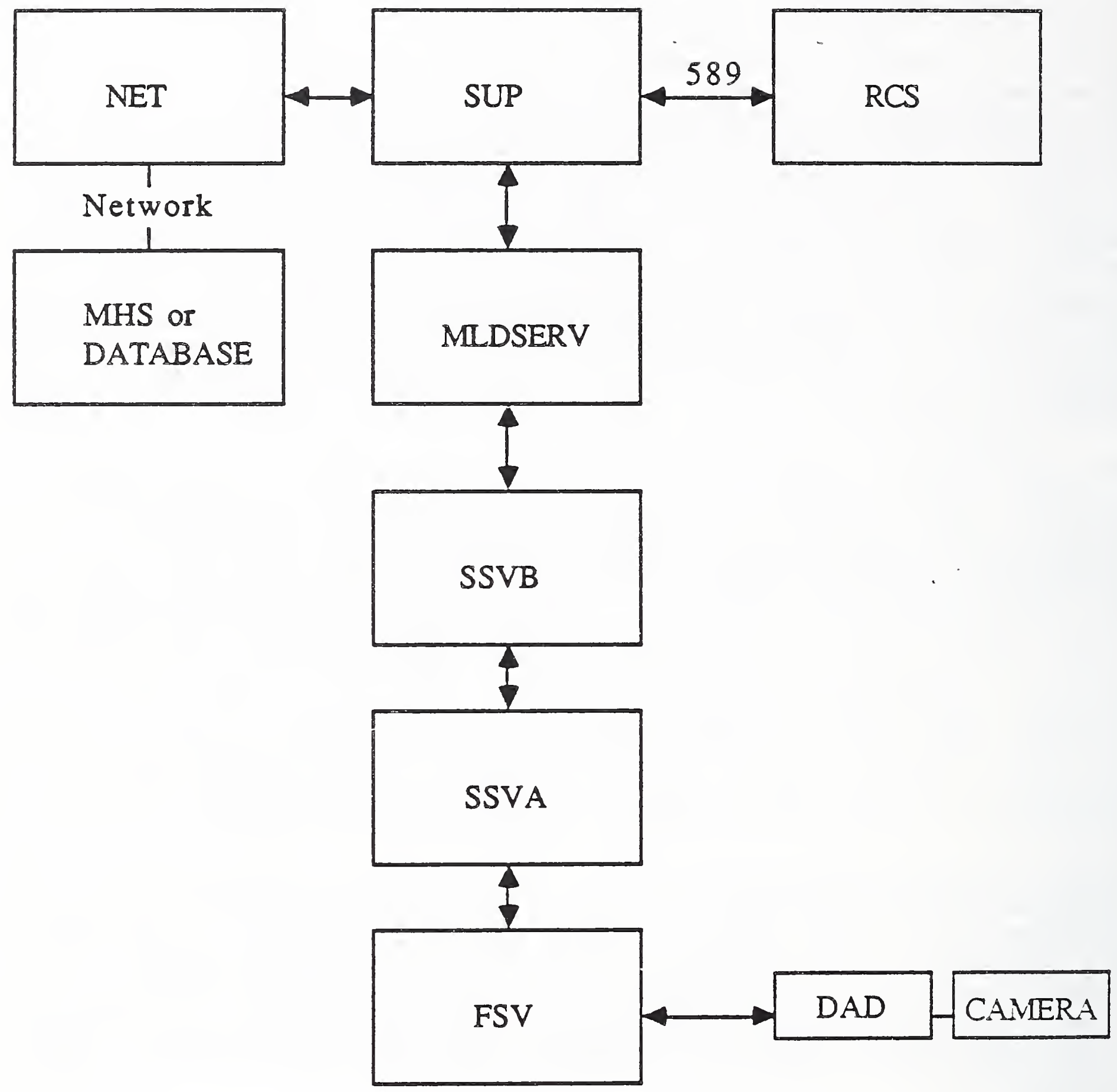

Figure 3. NBS Vision System 
NBS Vision System

A question which describes a default tray configuration is then posed to the vision system. Upon completion of the recognition task, a status report is sent to MHWS in the status mailbox. 


\section{FUTURE PLANS}

Future plans include expanding the system software to make it more versatile. The MHWS interface will be able to send UVA protocol commands and to interpret more sophisticated status messages. MHWS will be able to send commands to establish the configuration between itself and the vision system. Vision will also utilize its capability to notify MHWS of the status of the verfication request and to detail either reasons for failure or to update part locations.

The database interface will be used to extract information relative to any particular tray and to store updated information produced by the vision system. An MHWS command will be decomposed to obtain the necessary keys for vision to access a tray contents report and a tray definition report from the database. Information from these data reports will be used to construct a tray verification question. The updated part positions will be posted to the database. 
NBS Vision System

\section{REFERENCES}

[1] Nanzetta, P. and Rippey, W., "Integration of the AMRF", [To Come ]. 


\section{APPENDIX A. DATA STRUCTURES}

\section{RCS COMMUNICATIONS}

Following are the input and output structures defined between RCS and SUP:

\# define CMDLNGTH 500

\# define RSPSLENGTH 500
/* length of the command data field */

/* length of the response data field */

struct inbuffer

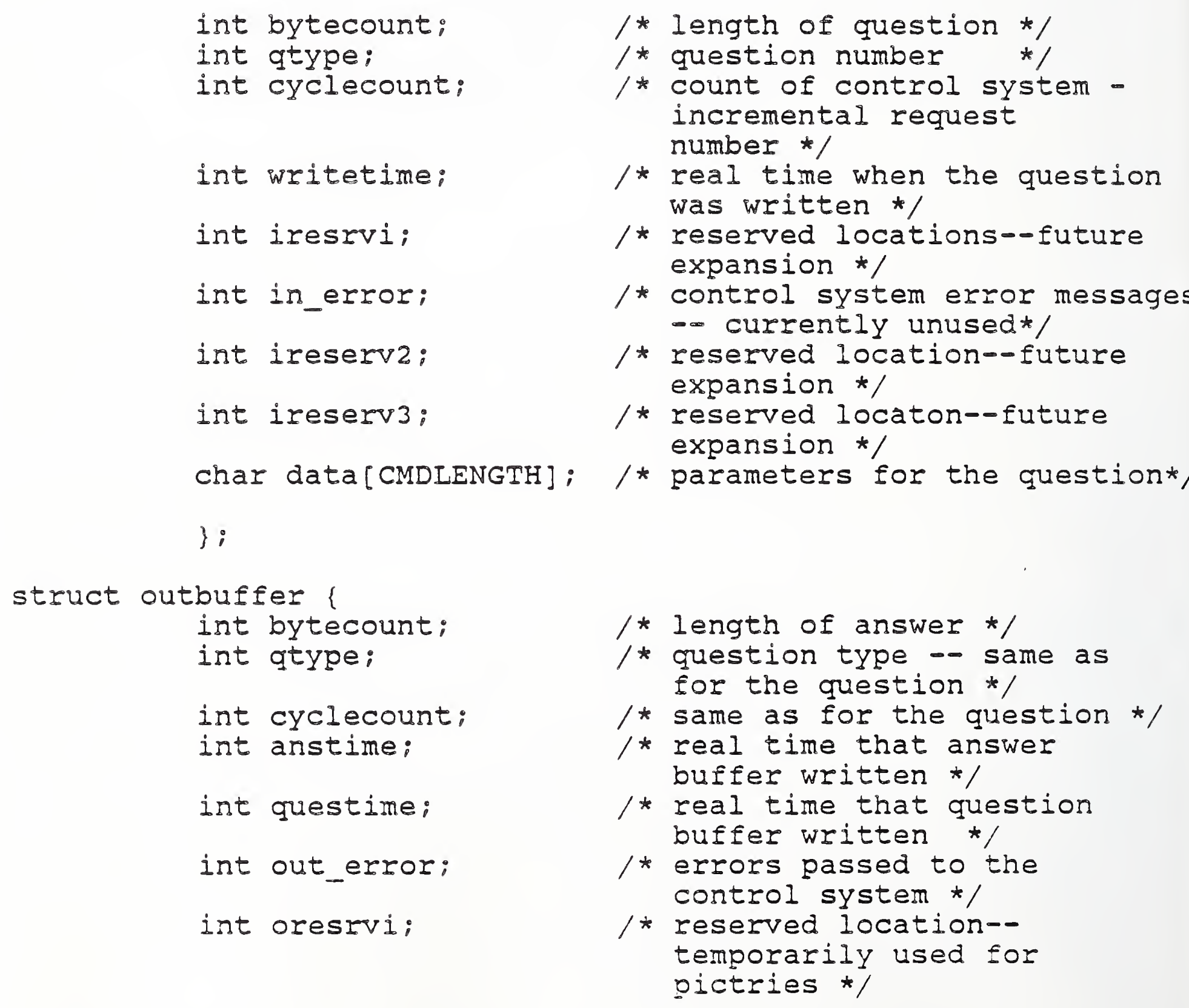


int oresrv2;

char data[RSPSELENGTH] \};

struct prog589
char cmabyte;
char statusbyte:
char chpl;
char chp2;
char chp3;
char chp4;
char devnumi;
char dtypel;
char mempti;
char mempt2;
char mempt3;
char mempt4;
char devnum2;
char dtyppe2;
char mempt5:
char mempt6;
char mempt7;
char mempts;
char bcounti;
char bcount2;
char bcount3;

\};

2. NETWORK COMMUNICATIONS

\subsection{Mailbox Transfers}

struct mgrm int $\mathrm{mg}$ seq; int mg-len;

char mḡ_tXt[MAXIGM];

\}

st

2.2 Database Transactions struct work_element_def \{

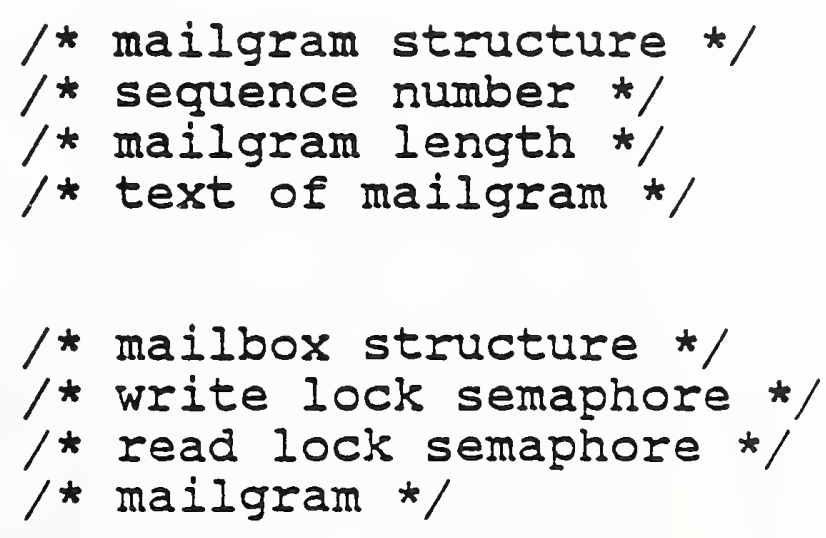

* Description of bytes
used for communication
between RCS and vision */

/* reserved location */ 


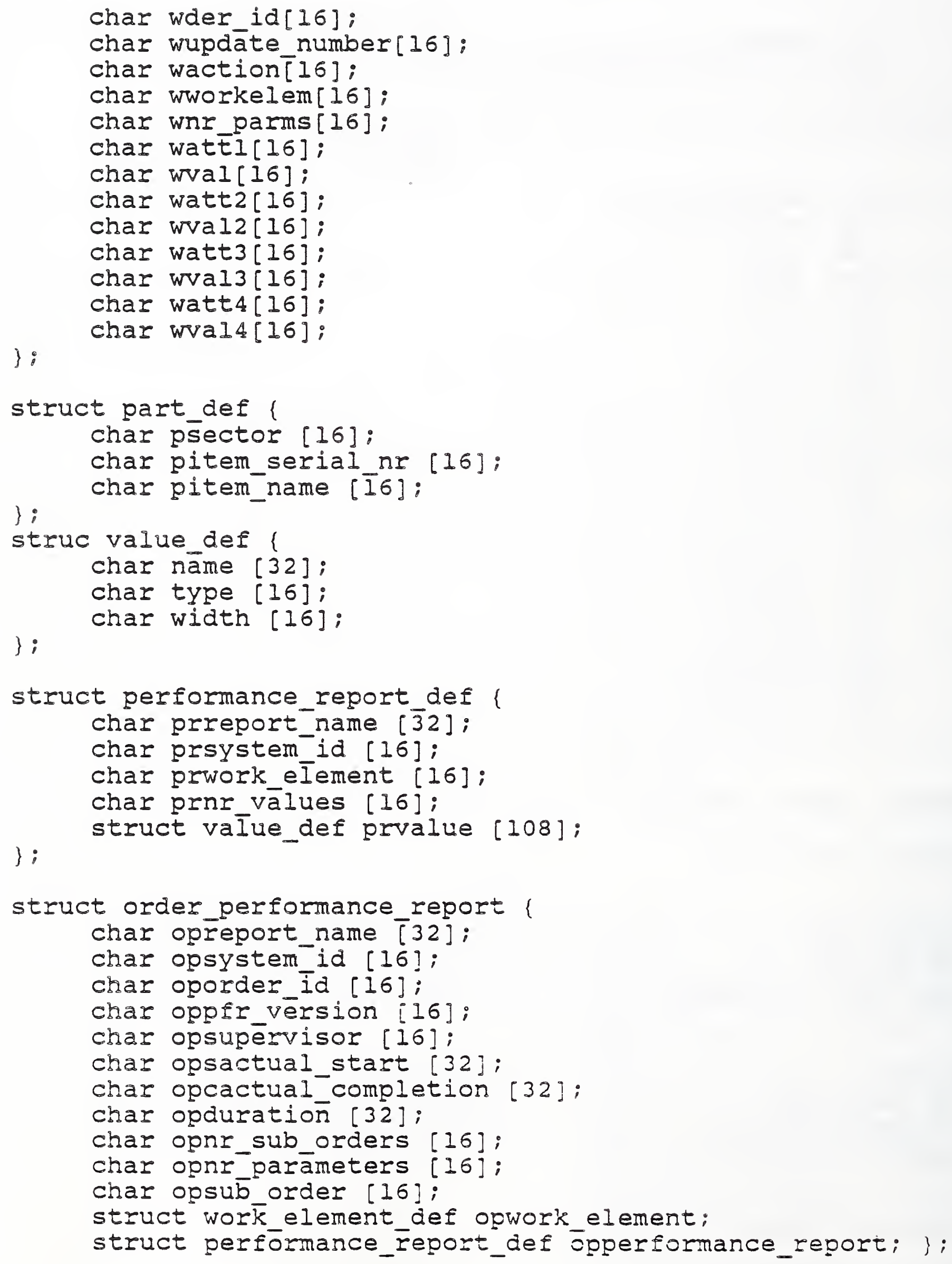


struct date time \{ char dFeport name [32]:

char dcurrent_time [32];

\} ;

struct sector_def

char ssector [16]:

char sx_offset [8];

char sy_offset [8]:

char sz_offset [8]:

char sx_dimension [8];

char sy-dimension [8];

char sz_dimension [8];

\} :

struct tray definition_report \{ char tareport_name [32]; char tditem sübtype [32]; char tanr_locations [16]; struct sector_def tdsector [9]:

\} ;

struct tray_contents_report 1 char tcreport name [32]; char tctray_id [16]; char tcitm subtype [16]; char tctraȳ_clear [16]: char tcnr Iocations [16]:

\} ; struct part_def tcpartpos[9];

typedef struct Transaction ByteType iEype transaction;

char itransaction id [4];

char old_user_id $[\overline{8}]$;

char old transaction_id[4];

char *dmI_string;

struct Transaction *nnext;

\}_Transactioñ;

typedef struct \{

struct i

SeqType ncommand_number:

\} fixed_segment:

char userid [8];

-Transaction *dtransaction;

\} DataserverCommand; 
\}:

struct work_element_def cwork_element;

struct status_message \{ char smessage_id [4];

int slen;

int snum:

char stime [12];

int secho_len;

int strns len:

int sder Ien:

int srce len:

int scomño:

char scomtime [12];

int strns_statup;

char strns_keywd [16];

int strns parament;

int sder_num:

struct work_element_def swork_element;

; ;

3. COMMUNICATIONS BETWEEN MIDSERV AND SUP

3.I Data Structures for the Questions:

struct $q 1\}$

unsigned int qlobjid;

int qlrange;

\}:

struct q2

unsigned int q2objid:

int q2range;

\} ;

struct q3

unsigned int q3objid;

int q3range;

int $q 3 \cos x$;

int $q 3 \operatorname{cosy}$;

int $q 3 \cos z$;

\} ;

struct $q 4$

int $q 40 b j i d$;

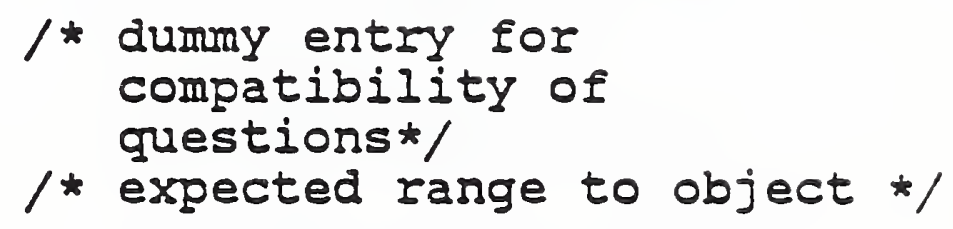

1 * dummy entry for compatibility of questions*/

/* expected range to object */

/* expected object name */

/* expected range to object*/

/* expected object rame */

/* expected range to object */

$/$ * directed cosines of normal to surface */ 
\};

struct 95 i

int sector:

float origin[2]:

float size[2];

char identifier[16]:

int flag:

unsigned int q5error:

float qg, qy, qz:

float qorientation:

\}$:$

3.2 Structures for the Answers:

struct al ?

int aldumm [3]:

int alcosx;

int alcosy:

int x_blob_centroid;

int y_blob_centroid:

\};

struct a2

unsigned int arobjid:

int confidence:

int best_obj_fit;

int $z$ _axis_ränge;

int roll_angle;
$1 *$ sector number */

$/ * x, y$ position of sector origin */

$I^{*} x, y$ position of sector size */

/* part identifier */

$/ *$ part verified?

'no' =0 'yes' or 'no part' $=1 * 1$

$/$ * reason for failure */

$\gamma^{*}$ position of part centroid */
$I^{*}$ dummy entry for answer compatability */

$/ *$ cos of $x$ comporent of angle between lens axis and line to centroid of blob */

/ $\cos$ of $y$ component of angle between lens axis and line to centroid of blob */

I* observed \& coordinate of centroid if height was given, otherwise 0 */

/* observed y coordinate of centroid if height was given, otherwise 0 */

/* expected object name */

/* confidence in

identification (computed as difference between between expected and computed distances */

/* actual best id */

$/ *$ observed range to object $z$ axis */

$I^{*}$ observed roll angle of 
NBS Vision System

int x_pos_centroid:

int y_pos_centroid; object */

$I^{*}$ observed $x$ coordinate of centroid*/

/* observed y coordinate of centroia*/

\} ;

struct a3 \{

unsigned int a $30 b j i d$;

int a3dumny[2];

int obs_range;

int cosx_obs:

int cosy_obs;

\} ;
/* expected object name */

/* compatibility with other answers* /

$I^{*}$ computed range to object *)

/* directed cosines nomal to surface */

struct a 4

float led[4][2];

/* centroids of 4 leds*/

\};

struct as

int sector:

float origin[2];

float size[2];

int flag;

unsigned int q5error;

float $q x, q y, q z$;

float qorientation;

\};

struct model \{

char *modname;

float modarea;

float modperim;

float modaxis;

float longside;

float shortside;

float modheight;

float bounds $[6]$;
$/^{*}$ sector number $* /$
$/ *$ x $x$ position of sector origin */

/* part identifier */

${ }^{*} *$ part verified?

'no' $=0$ 'yes' or 'no

part' $=1$ * 1

1 * reason for failure */

$/ *$ models used in server */

$1 *$ ASCII name */

/* area of object in square mm */

$1 *$ perimeter in $\operatorname{mm} * /$

$/ *$ principal axis

calculated */

$/ *$ length of long side - in $\mathrm{mm} * /$

$/ *$ length of short side */

$I^{*}$ bounds on range of 


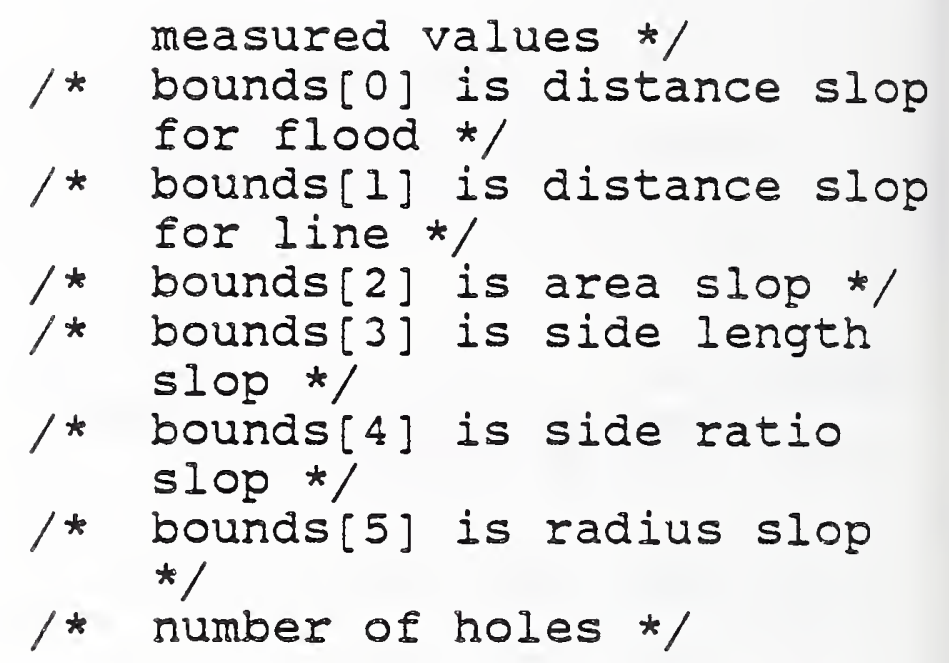

3.3 Model Data for the objects

struct model proto []$=\{$ /* box bottom */

/* box top */

$/ * \operatorname{flag} * /$

"flag",

/ cylinder */

"cylinder",

"box top",

"box bottom"
7137.0,
0.0 ,
74.93
1.1,
1.1,
0.1 ,
0 ,

7137.0 ,

0.0 ,

74.93 ,

1.1,

1.1,

0.1 ,

0 , 7679.0,

0.0 ,

24.13 ,

1.1 ,

1.08 ,

1.1,

469.97 ,

0.0 ,

50.3 ,

1.1,

1.25 ,

0.1 .

1 ,

1516.5 ,

0.0 ,

43.9 ,
340.0 , 95.25 , 36.83 ,

1.1,

1.06 ,

1.1 ,

340.0 , 95.25 ,

17.78 ,

1.1,

1.06 ,

1.1,

350.5 ,

87.63

1.1 ,

1.15,

0.1 ,

0 ,

158.0 ,

50.3 ,

25.4 ,

1. 1 ,

1.08 ,

I.1,

138.05 ,

43.9 ,

25.4 , 
$/ * \operatorname{dog}$

$/ *$ ring*/

/* turning adapter */ "thold",

/* boring adapter */ "bhold",

/* pipe flange 205 */

$$
\text { "ring", }
$$

/* pipe flange 207 */

"£1207-b",

"dogola",

$$
\text { ". }
$$

"£1205-b",

/* pipe flange 209

$\begin{array}{rr}1.1, & 1.1, \\ 1.15, & 1.08, \\ 0.1, & 1.1, \\ 0, & \\ 3225.8, & 266.75, \\ 0.0, & 101.6, \\ 31.75, & 31.75, \\ 1.1, & 1.1, \\ 1.15, & 1.2, \\ 0.22, & 1.1, \\ 0, & \ldots 1 \\ 235.7, & 62.86, \\ 0.0, & 0.0, \\ 0.0, & 0.0, \\ 1.1, & 1.1, \\ 1.2, & 0.0, \\ 0.0, & 0.0, \\ 0, & \end{array}$

$5741.924, \quad 307.848$, $0.0, \quad 90.424$,

63.5, 63.5,

38.1, 1.1,

1.1, 1.1,

$1.09, \quad 0.08$,

1.1,

5741.924307 .848 ,

$0.0, \quad 90.424$,

63.5, 50.8,

1.1, 1.1,

$1.1,1.09$,

$0.08,1.1$,

0 ,

$2199.092, \quad 195.58$,

$$
\begin{array}{cc}
0.0, & 62.738, \\
35.1, & 23.0, \\
1.1, & 1.1, \\
1.1, & 1.1, \\
1.09, & 0.08,
\end{array}
$$

1.1 ,

$4143.167,266.71$, $0.0,84.074$, 49.28, 27.0, 1.1, 1.1, 1.1, 1.09, $0.08,1.1$,

0 ,

$7646.760, \quad 358.65$, $0.0, \quad 109.47$, 
/* plunger bracket */ "brack-b",

/* IGES test part */

/* valve body */

${ }^{*}$ pen set base

$$
\begin{aligned}
& 69.85, \\
& 1.1, \\
& 1.1, \\
& 0.08, \\
& 0,
\end{aligned}
$$
1862.667 , 0.0 , 34.93 ,

1.1,

1.1,

1.1,

1.1,

"iges-b" ,

"valvem-b",

"penset", 6541.77 ,

0.0,

50.5 ,

1.1,

1.1,

0.08 ,

0 ,

18749.59 ,

0.0 ,

114.3 ,

I.I,

1.1 ,

0.08 ,

0 , 13200.0 ,

$$
\begin{aligned}
& 42.9 \\
& 1.1 \\
& 1.09 \\
& 1.1
\end{aligned}
$$

252.77 , 72.974 , 40.46 ,

1.1,

1.2 ,

0.08 , 0.0 ,

75.0 ,

1.1,

1.1,

0.1 ,

0 ,

03.21

0.0 ,

63.5 ,

1.1,

1.1,

0.1 ,

0 ,

/* 15: hinge block */ "hinge block", 3654.0, 0.0,

58.0,

1.1,

1.1,

0.1 ,

0 ,
242.0
63.0,
32.0 ,
1.1,
1.05,
1.2 ,

312.93

92.964

50.8,

1.1,

1.06,

1.2,

/ 16: Iink head */ "Iink head", 1575.0

163.0, 


\section{/* 17: Iink bar */}

"link bar",

"block",

/* 19: $\operatorname{dog} * /$

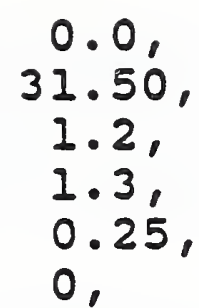

1616.0 , 0.0 ,

16.0,

1.2 ,

1.25 ,

0.25 ,

0 ,

6750.0 ,

0.0 ,

50.0 ,

1.1,

1.1,

0.0 ,

0 ,

"dog",

4066.0 ,
50.0 ,

50.50 ,

1.1 ,

1.06 ,

1.1,

234.0 , 101.0,

16.0,

1.1,

1.20 ,

1.1,

37.00 ,

135.0 ,

50.0,

1.1,

1.06,

1.1,

0.0

38.0 ,

1.2,

1.3,

0.25 ,

0 ,

$/ *$ nuIl model to end $* / " 1,0.0,0.0,0.0,0.0,0.0,0.0,0.0$, $0.0,0.0,0.0,0.0,0.0,0$,

\}:

4. FIIE COMMUNICATIONS

struct filebuf \{

$\begin{array}{ll}\text { char } & \begin{array}{l}\text { filename[16]; } \\ \text { chat }\end{array} \\ \text { uchar } & \text { prevuser; } \\ \text { uchar } & \text { otheruser; } \\ \text { char } & \text { owner } \\ \text { unit } & \text { otherusers; } \\ \text { uchar } & \text { multiple; }\end{array}$

/* one structure for each file*/

/* open or closed (this flag is internal to program) */

/* file is opened */

$1^{*}$ userid of other process using this file */

$/^{*}=1$ if this processor "owns" file */

/* Each bit is a user. Bit 15 set means this is the owner */

$1^{*}$ True says more than one reader at a time may access file */ 


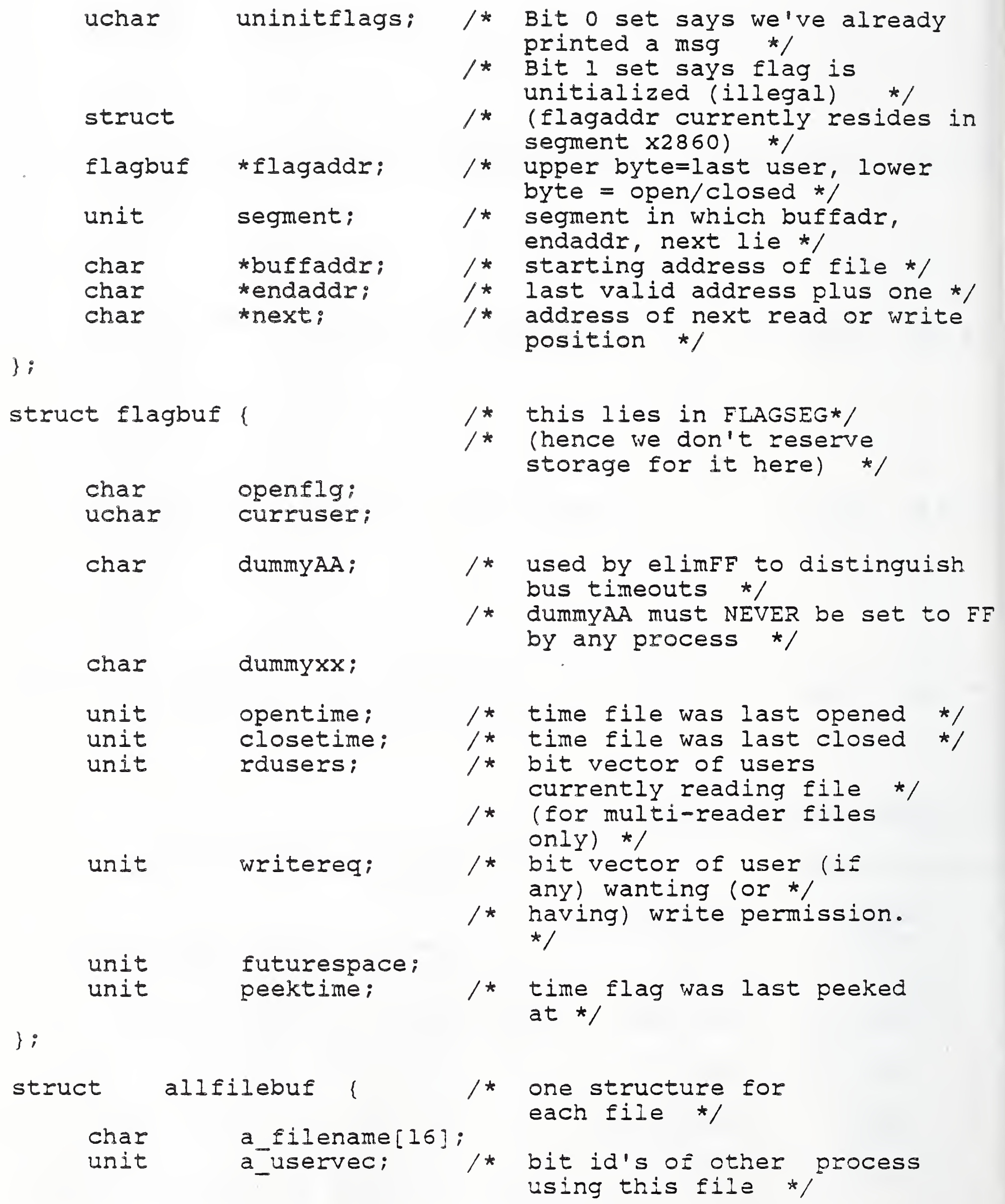


unit a_owner; $\begin{aligned} / & \text { bit id of "owner" of file */ } \\ & \text { (flagaddr currently } \\ & \text { resides in } \times 2860)\end{aligned}$

struct flagbuf *a_flagaddr:

/* address of system flagbuf for this file */

unit a_segment; $\quad /$ segment in which buffadr,

endaddr, next lie $* /$

char *a_buffaddr; / * starting address of file */

char *a_endaddr; $/$ * last valid address plus one */

int a_multiple;

/* True means more than one reader at a time may access file */

int a_dummy2;

/* make allfilebuf an even 32

bytes */

\} ;

5. OBJECT DESCRIPTION

struct ssvaobj \{

long8 aarea, axcntr;

long8 aycntr,am20,amli;

long8 di, d2;

\#ifdef MOMENTS

longs ammlo, ammol, amm20,

amm02, amml1, amm21, amml2,

\#endif $\operatorname{amm} 03, \operatorname{amm} 30$;

int ast_list;

int aparent, achild,

asibling, acolor, aximin,

axmax, aymin, aymax;

int comp;

int ah_area, al area;

struct frame *aframe;

int awhole;

\} ;

struct objects \{

double area;

double xontr, yontr;
/* the structure received

from ssva */

/* scratchpad for ssva */ 
double m20, mIl:

double mmio, mol, mm20,

mm02, mmli, $\operatorname{mm} 21, \operatorname{mmo3}, \operatorname{mm} 30$;

float perim;

unsigned int equation:

int holes;

unsigned int olinks[2]:

int future;

int past;

int odescription:

int ox2d:

int oy $2 d$;

float oaxis2d;

int ox $3 \mathrm{~d}$;

int oy $3 d$;

int oz $3 d$;

int oyaw;

int opitch:

int oroll;

unsigned int st_list:

unsigned int s_corn, e_corn:

unsigned int parent, child, sibling:

int color:

int xmin, xmax, ymin, ymax;

unsigned int h_area, I_area;

int ocoordsys:

struct frame *oframe;

int otype;

int oname;

int opart_of:
/* object moments */

/* index to length of boundary (not including holes) */

/* index of structure containing surface equation */

/* number of holes */

/* links to other objects */

/* link to next frame */

/ link to previous frame */

/* further information about the object */

/* 2d position: $x, y * /$

/ 2d principal axis

(radians) */

/* 3d position: $x, y, z * /$

/* 3d position: yaw, pitch, roll (degrees) */

/* start address of edge list */

start and end

pointers for corners */

$/ *$ links in tree of components */

$1 *$ object or hole */

/ bounding rectangle */

/* links to next largest and next smallest components */

/* which coordinate system is used for position */

/* pointer to header structure for picture */

$l^{*}$ object type or number */

/* name from model database*/

/* which object it belongs to * 
int oconfidence;

int whole;
$I^{*}$ is this really the right object? */

/* I if completely processed by ssva, 0 otherwise */

\section{; :}

5.1 Structures for Features in Database:

struct feature \{

double surfegn [4];

int fx2d;

int fy2d;

float faxis2d;

int fx3d;

int fy3d;

int fz3di

int fyaw;

int fpitch;

int froll:

unsigned int flinks[2];

unsigned int fdescription;

int fedgenum;

unsigned int fcomponent[3];

int fwindow [4];

int ftype;

int fname:

struct frame *fframe;

unsigned int fpart_of;

int fconfidence;

int fcoordsys;
/* surface equation if feature is a surface */

/* 2d position: $x, y$ */

/* 2d angle (radians) */

/* 3d position: $x, y, z$, yaw, pitch, rol1 */

/* 3d position: yaw, pitch, roli (degrees) $\star /$

/* links to other features * 1

/* further information about the feature */

${ }^{*}$ number of edge points if edge feature */

1 * which components(s) it belongs to */

/* e.g., flood blobs */

$/ *$ coords of window it was detected in */

1 * feature type or number */ name from model database *I

$I^{*}$ pointer to header structure for picture */

$I^{*}$ which object it belongs to in ssvb */

$1^{*}$ is this really the right feature? */

$/^{*}$ which coordinate system is used for position */ 
struct cheby

int firstx;

int lastx:

double coeffa;

double coeffb:

double coeffc:

double coeffbb:

double coeffcc:

double cerros:

unsigned int *cequation;

unsigned int cnumfeats;

int chebfeats;

int nextcheb:

struct frame *cpicture;
/* The Chebyshev

coefficients, errors, and line endpoints */

/* image coordinate start and end points of segment */

/* coefficients of polynomial *

/* origin is at firstx */

/* (alternate coefficients) */

$f^{*}$ origin is at midpoint of curve */

/* fitting error */

$/ *$ equation of surface

$/$ * number of features */

$/ *$ pointer to corners of segment $* /$

$I^{*}$ next structure of this type * 1

/* pointer to frame info */

/* Chebyshev structures */

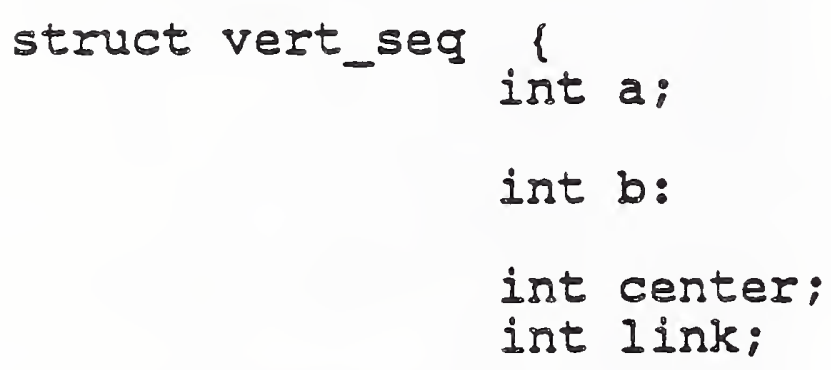
/* dark to light transition */
/* light to dark transition */
$/ *$ center $=(a+b) / 2 * /$
$/ *$ pointer to next segment */

\};

struct start_list

int pointer; /* pointer to start seg */ int column; $/ *$ column index of segment */ \}:

5.2 Structure for Frame-Dependent Information:

struct frame \{

unsigned int ferrorstat; /* system errors */ unsigned int ftod;

$/$ * time of day when picture was taken */ 
unsigned int fsequence; /* unsigned int fpictype; /* unsigned int fnumnodes; /* unsigned int ffirstnode: /* unsigned int frummatches;/*

unsigned int fnumedges: $/^{*}$ unsigned int ftabentries;/* sequence number for

$$
\text { picture */ }
$$

the picture that was requested */

number of data entries found */ pointer to first node */ number of matches whith expectations */

/* used to be fnumfeats still is for old system */

number of edges */ number of table entries */

\section{了:}

6. PICTURE COMMAND STRUCTURE

\section{I Structure for Commands Passed Between MIDI and SSVA:}

struct pictype \{

$$
\text { unsigned int time: }
$$

int cambeight;

unsigned int bwindow;

unsigned int lwindow;

unsigned int rwindow;

unsigned int twindow;

unsigned int flasht;

unsigned int flashval;

unsigned int threshold;

unsigned int refadd:

unsigned int dadmode;
$I^{*}$ marker for current request */

/* height of camera or line flash above object */

$1^{*}$ bottom position of window for camera */

$/ *$ left window $* /$

1 * right window */

$/ *$ top window */

/* type of flash (line of flood) */

flash intensity */

threshold */

additive factor for

reference frame (0-255)

Mode 0 *

1 * mode of using the dad box: 0 = pixel-by-pixel threshold */

/* (two pictures required),

$I$ = "normal" single

threshold mode */ 
unsigned int reflshval;

unsigned int reflasht;

unsigned int opmode;

unsigned int flashtest;

long int campos [6]:

unsigned char picstatus;

unsigned char numwind; unsigned char startwind[10]; /*

unsinged chat endwind [10]: unsigned int data_mode; unsigned int d_type;

unsigned int b_w_filter;

unsigned int w_b_filter:
/* duration of flash for reference frame */

$/ *$ dadmode 0 only, $0-15$ */

$/^{*}$ flash type of reference frame inage */

/* mode of operation: $1=$ operate default if no commands from above */

/* 0 = wait for new commands before proceeding */

/* flag for testing that flash fired. $0=$ ignore flash test */

$I^{*} \quad l=$ perform flash test */ 1 * 6 words for the position of the camera *)

$I^{*}$ whether a normal request $(0)$ or a number of frames *)

/* to tell fsv to process for range in the given window */

number of windows */

up to 10 windows:

defined by start and end */

/* $0=$ read in 16 transitions; $1=$ read in run centers */

1 * used only when data mode is 1. 0 = integer + fraction returned */

$/ * 1=16$ center points returned $* /$

/* noise filter for black to white transitions $(0,1$, 2,3 , pixels) */

$1 *$ noise filter for white to black transitions $(0,4$, 8, 12) */ 
NBS Vision System

APPENDIX B. DEBUGGING OPTIONS

Interactive debugging options are provided for viewing intermediate results and for running the system in a simulated mode. To enable this mode, press any character on the supervisor keyboard while the system is running. A menu will appear on the screen with nine choices. They are explained in further detail below:

1. Vision system status - displays current vision system status with respect to presence of external systems.

2. Command stack dump - shows the contents of the vision command queue.

3. Command/answer history stack dump - shows approximately the 10 most recent question/answer pairs.

4. Change conditional printout variables - permits the user to change the level of detail in the printouts.

5. Reduce command stack and history stack - flushes the queue so that the control system does not have to wait for all of the queued commands to be executed.

6. Modify default thresholds - interactively changes the threshold for any object for either flood flash or line flash images.

7. Modify default picture - allows the user to interactively create a question and send it to either RCS or MrWW .

8. Change DADBOX mode - determines the mode in which the DAD framebuffer will be used.

9. Set up automatic sequence of questions - allows the user to specify a sequence of simulated commands to be posed to the vision system.

For commands 2 and 3 above, the format of the data is in unsigned decimal bytes. The bytes are in the protocol of input/output between the vision and control systems. Refer to Appendix A for the data structure format.

For command 4 , the SUP printouts can be interactively changed to suit the user's needs. This provides a tradeoff between processing speed and debugging power. When command 4 is invoked, 
each debug variable is printed out along with its current value. There are print statements in the program which are conditional on one of these debug variables; a "o", ignores the printout while a "I" permits it. The following debug variables are currently available:

1. ptrace - prints out a trace showing the order in which functions are called in the program.

2. debug - prints out almost everything.

3. debugm - prints out the returns in the main loop of the program.

4. debugvc - prints out the returns in the vision command section, a major part of the supervisor program.

5. debug589 - prints out useful information about the interaction between the control system and the vision system.

6. debugvel - prints out lots of information concerning the acquisition of errors from the vision levels during picture processing.

7. debugve2 - prints out the errors from the vision levels during picture processing.

8. debugh - enables the history stack so that the most recent 10 commands and their answers can be stored for future use.

9. debugl - enables the system to terminate when a drastic error occurs.

Another interactive feature, command 5, allows the command and history queues to be reduced so that they contain only the current command. This option is useful in cases where suP has forced many simulated commands onto the queue before the control system initialized the 589 board. This option allows the control system's command to be processed immediately. Also, when the vision system is being operated in debug mode, this option allows different vision commands to be executed quickly.

Command 6 permits the user to change default thresholds for each part. When this command is activated, the first question chooses the type of flash, line or flood. The second question chooses 
the part type. The user must enter the number of the part as it appears in the data structure, model (see Appendix A). The third question is the request for a new threshold value.

With command 7, the user can interactively send a question to the vision system. There are 5 choices of questions. Note that all flash values range between $1-10$, and all range values and sector sizes are in millimeters. Question 1 calculates the centroid of one object in the field of view. Question 2 verifies that the part in the image is the expected part and also calculates its position and orientation. Question 3 calculates the range to the part in the image. Question 4 computes the centroid of four I.E.D.S in the field of view. This question is available but is not used in the AMRF. Question 5 verifies the occurrence of a part in a specified sector of a tray and also returns that part's position and orientation.

The next interactive feature, command 8 , involves setting the thresholding used by the DAD hardware.

The last command, 9, allows the user to specify which sequence of questions will be posed to the vision system in simulated command mode. 
NBS-114A (REV. 2.8C)

U.S. DEPT. OF COMA.

BIBLIOGRAPHIC DATA

SHEET (See instructions) 1. PUBLICATION OR
REPORT NO.

NBSIR $87-3684$
2. Performing Organ. Report Nof 3. Publication Date

December 1987

4. TITLE AND SUBTITLE

The NBS Vision System in the AMRF.

\section{AUTHOR(S)}

Marilyn Nashman and Karen Chaconas

6. PERFORMING ORGANIZATION (If joint or other thon NBS。 see instructions)

NATIONAL BUREAU OF STANDARDS

U.S. DEPARTMENT OF COMMERCE

GAITHERSBURG, MD 20899

7. Contrace/ Grane No.

9. SPONSORING ORGANIZATION NAME AND COMPLETE ADDRESS (Street, City, Stote, ZIP)

National Bureau of Standards

Route $\left.\right|^{2} 70$ and Quince Orchard Road

Gaithersburg, MD 20899

10. SUPPLEMENTA.RY NOTES

Document describes a computer program; SF-185, FIPS Sotiware Summary, is attached.

11. A BSTRACT (A 200-word or less foctual summary of most significant information. If document includes a significant biblicgrophy or literoture survey. mention it here)

This document describes the NBS Vision System in the AMRF. It discusses the objectives of the vision system and its applications in the factory environment. Since the vision system is a multi-processor system, each process is described according to its position in the vision hierarchy as well as to its particular logical and computational functions. The unique hardware used is discussed and its capabilities described. In addition, a guide to operations is included: This contains step-by-step directions for "bringing up" the system in either stand-alone mode or integrated mode. The interfaces between the individual processes of the vision system, as well as the interfaces between the vision system and other AMRF systems, are described. Finally, appendices are included which describe data structures, and debugging features.

12. KEY WORDS (Six to twelve entries; olphabetical order: capitalize only proper names: and separate key words by semicolons) Automated Manufacturing Research Facility; hierarchical design; Material Handling Workstation; multi-processor system; Realtime Control system; realtime vision system.

13. AVAILABILITY

X. Unlimited

For Official Distribution. Do Nor Release to NTIS

Order From Suder:ntendent of Documents, U.S. Government Printing Office, Washington. D.C. 20402.

X Order From National Technical Information Service (NTIS), Soringfield, VA. 22161
14. NO. OF PRINTED PAGES 48

15. Price

$\$ 11.95$ 
. 
